\title{
What is life made of?
}

\author{
A. Chopra \\ Planetary Science Institute, Research School of Astronomy and Astrophysics \& Research School of \\ Earth Sciences, The Australian National University, Acton ACT 0200, Canberra, Australia
}

As we begin to understand the origin and evolution of terrestrial life and investigate the possibility of extraterrestrial life, the need to scientifically approach fundamental questions such as 'What is life?' increases. In beginning to answer such questions we can look at the ingredients of terrestrial life. Here we present an overview of our understanding of the composition of terrestrial life. At the level of chemical elements, the major ingredients are carbon, hydrogen, oxygen, nitrogen, sulphur and phosphorus along with trace amounts of elements like sodium, potassium, iron and copper.

Why terrestrial life is made of this particular set of elements is not clear. Here we present the correlation between the elemental composition of life (humans and bacteria) and sea water. Elements essential to life (e.g. carbon and sodium) are more abundant in sea water than nonessential elements (e.g. tungsten or lead) suggesting that life utilises elements that are easily obtained from its environment.

We quantify differences in elemental abundances in life, relative to sea water, and attempt to interpret them in terms of chemical constraints on metabolic activities and harnessing energy. We discuss how future investigations could further our understanding of the origins and evolution of life on Earth.

While natural philosophers ${ }^{1}$ believed all matter to be made of four elements: fire, air, water and earth, we now recognise that all matter in the universe is made of atoms of chemical elements. ${ }^{2}$

The first chapter of [1] is titled: "A chemical analysis of the elements which are found to exist in the human frame - thus showing the natural origin of man." It is perhaps one of the earliest records where one has examined the composition of life in terms of chemical elements. De Courcey mentions that vegetable and animal matter are essentially composed of carbon, hydrogen, oxygen and nitrogen in addition to sulphur, phosphorus, iron and small quantities of "saline matter" (for example sodium and potassium).

$\mathrm{He}$ goes on to give us relative proportions of these elements in "fibrin - the lean part of animals, which is composed of 18 parts of carbon, 14 of hydrogen, 5 of oxygen and 3 of nitrogen." Upon examining the elements that make up the environment of the vegetables and animals, he concludes that the

\footnotetext{
${ }^{1}$ Empedocles proposed circa $450 \mathrm{BC}$ that the four classical "roots" known to pre-Socratic philosophers, made all the structures in the world. The term "elements (stoicheia)" was first used by Plato circa 360 BC in his dialogue Timaeus.

${ }^{2}$ Excluding the case of dark matter, whose nature and composition is still in question.
}

atmosphere, water and vegetables are composed of the "same elementary principles" and that "the Earth and the animal organization of the human frame is composed of the same agencies."

Today we have a much better knowledge of the composition of life and its environment, primarily due the suite of analytical techniques available to accurately measure the concentrations of elements in a sample.

The 'bulk' elements oxygen, carbon, hydrogen and nitrogen make up $96.8( \pm 0.1) \%^{3}$ of the mass of living matter and the remaining $3.2( \pm 0.1) \%{ }^{3}$ of the mass consists primarily of phosphorus and sulphur with $2.2( \pm 0.2) \%^{3}$ of the mass attributed to 'trace' elements like potassium, sodium, calcium, magnesium and other metals such as iron, copper and cobalt. Many of these trace elements are known to play a variety of biological roles as electrolytes and as enzyme cofactors and often the amounts and proportions of these elements are important in achieving successful functioning of the metabolic pathways in an organism. [5] have noted that "of the 90 kinetically stable elements that occur in nature,

\footnotetext{
${ }^{3}$ Values were derived from averaged elemental abundances in humans and bacteria, as presented by [3] and [4] respectively. The uncertainties reflect the deviation between the abundances in humans and bacteria.
} 


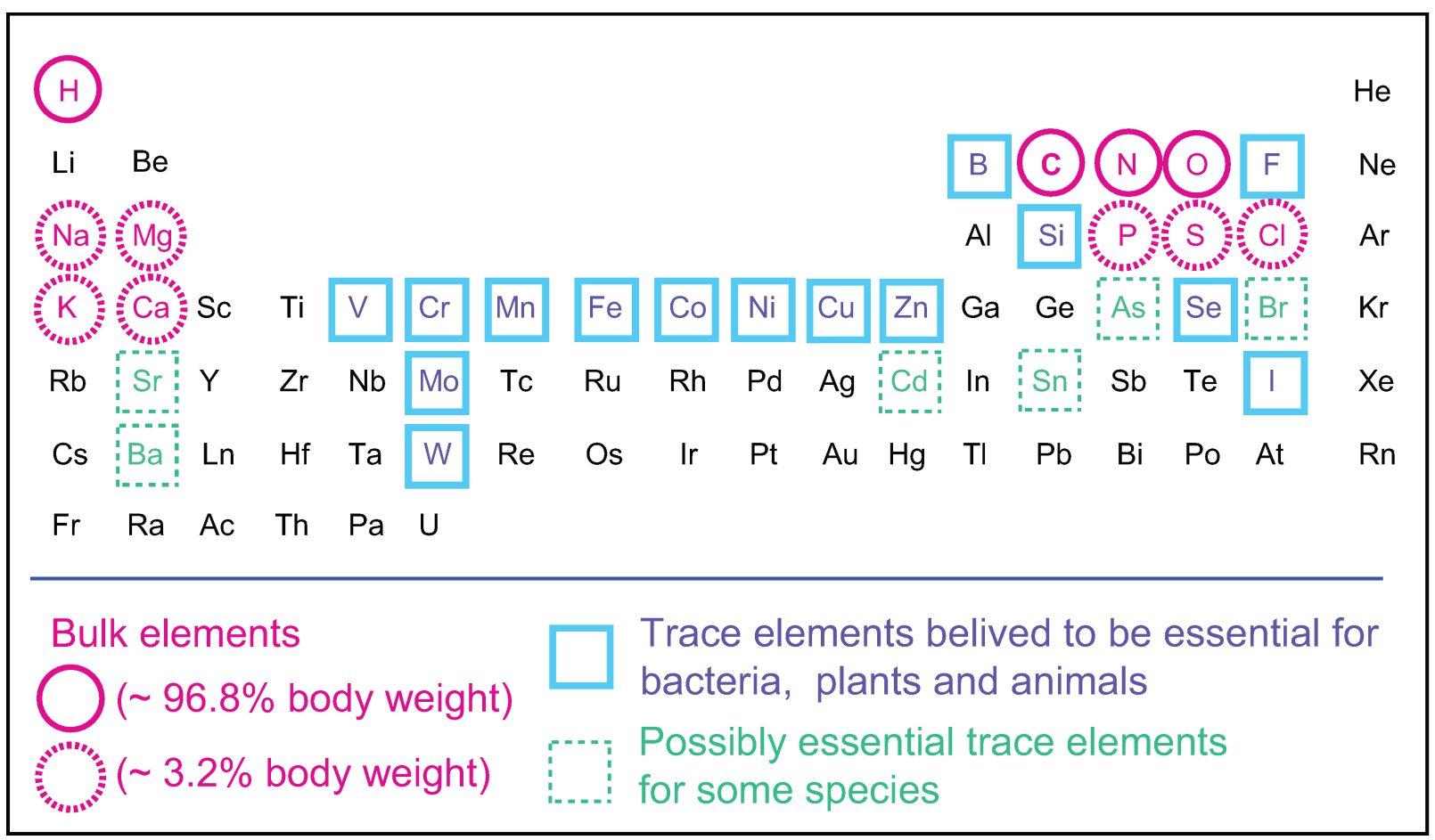

Fig. 1: The distribution of elements considered 'essential' for life in the periodic table. Adapted from [2].

about 36 are essential to some form of life (see Fig. 1)."

A number of authors including [5] and [6] have presented the relative proportions of the chemical elements in organisms like humans ${ }^{4}$ and bacteria ${ }^{5}$ and noted the similarity in their compositions. [6] has also noted that the elemental abundances in these species show strong correlation with the elemental abundances of sea water ${ }^{6}$. We have graphically illustrated the tabulated data from [6] in Fig. 2.

Life forms show many commonalities but also differ in their composition and usage of elements because of the different environments they live in. It is clear that any life form can only be made of the elements available to it from its environment. If element $\mathrm{X}$ is non-existent in an environment then any life in that environment will not be composed of element X. It is interesting to examine to what extent

\footnotetext{
${ }^{4}$ Human abundances from [3]. The study involved about 150 individuals.

${ }^{5}$ Bacteria abundances are from [4] and the values are representative of 7-40 species depending on the element.

${ }^{6}$ Sea water abundances are from [7] and based on empirical models that estimate the composition of normal surface sea water with a chlorinity of 19, which corresponds to approximately $34.5 \mathrm{~g}$ of salt per litre of solution.
}

the ingredients of life reflect the relative proportions of the elements in its environment. If we take sea water as an approximation to our diet then from Fig. 2 we can infer that ' we are what we eat' but also that ' we are not exactly what we eat.'

Although humans do not currently live in the oceans or drink sea water, the composition of our diet is much more similar to sea water than it is to the Earth' $\mathrm{s}$ crust. This is probably because the first $90 \%$ of our evolution, approximately 4 billion years ago to 400 million years ago, took place in sea water

The relatively high abundance of hydrogen and oxygen in life is primarily due to the water $\left(\mathrm{H}_{2} \mathrm{O}\right)$ that makes up about $65.9 \%$ (by number of atoms) of humans ${ }^{7}$ and about $83.6 \%$ (by number of atoms) for bacteria such as Escherichia coli [4]. The fewer number of data points for bacteria (25 elements) than for humans (58 elements) in Fig. 2 relates to the fact that bacteria have not been analysed to the same extent as humans have been for their elemental composition.

Unlike humans and bacteria, sea water is almost entirely composed of hydrogen and oxygen (hydrogen and oxygen in $\mathrm{H}_{2} \mathrm{O}$ makes up $99.1 \%$ of

\footnotetext{
${ }^{7}$ [8] suggests that human body water content can range from $56 \%$ for obese persons to $70 \%$ for a lean person and here we have used the mid-point value of $63 \%$ by mass.
} 

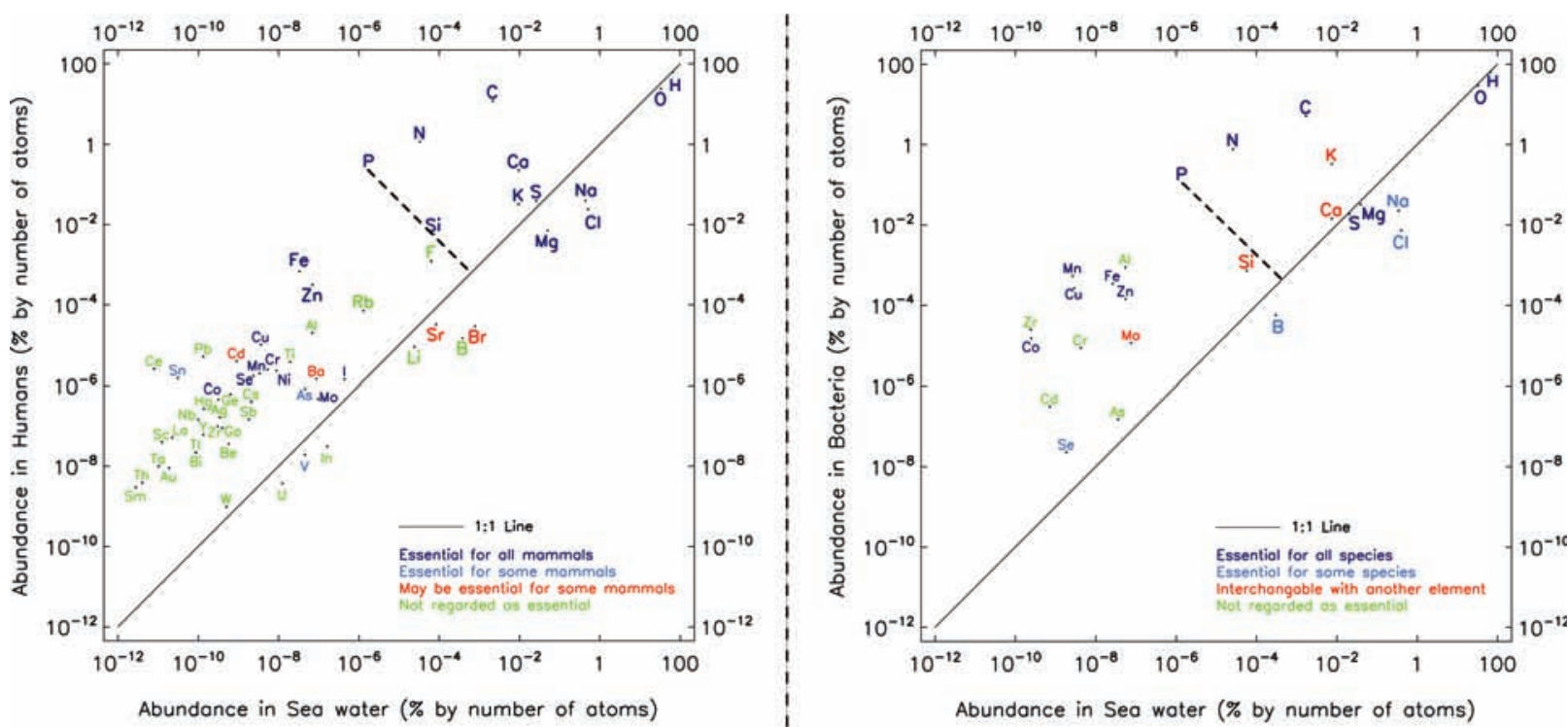

Fig. 2: Life vs. Sea water-The positive correlation between elemental abundances (by number of atoms) in humans and bacteria relative to ' boiled' sea water, which contains the same percentage of water (by number of atoms) as in the life form. Note the logarithmic scale and that although elements like sodium and boron appear to have similar deviations from the equilibrium value (as indicated by the 1:1 line), the absolute deviations are much greater. 'Essentiality' of elements for mammals and bacteria are colour coded as suggested by [6].

the atoms in normal surface sea water) with relatively negligible amounts of other elements that make up the 'sea salts'. To allow valid comparisons of the relative elemental abundances in life forms and the sea, we have reduced the quantity of $\mathrm{H}_{2} \mathrm{O}$ in sea water such that the percentage of water (by number of atoms) was equivalent in the life form and sea water. The treatment is analogous to 'boiling' sea water and concentrating the salts in sea water.

Due to the logarithmic axes used in Fig. 2, hydrogen and oxygen appear to have the least magnitude of deviation from the equilibrium value (as indicated by the 1:1 line) compared to other elements. However, the absolute deviations are much greater and account for the apparently large deviations in the opposite direction exhibited by the low concentration elements like cerium and lead.

It was surprising to find that if we 'boiled' sea water to the extent that no hydrogen and oxygen was present as $\mathrm{H}_{2} \mathrm{O}$, and similarly 'boiled' the life form (humans or bacteria) by reducing the water content to $0 \%$, we would still have a higher proportion of hydrogen and oxygen in seawater than in the life form. That is, there are more atoms of hydrogen and oxygen present as salts (such as bicarbonates and sulphates) and gases dissolved in sea water, than there are hydrogen and oxygen atoms in the nonwater component of humans and bacteria.
As expected, the most abundant elements in sea water after hydrogen and oxygen are chlorine and sodium that upon evaporation of sea water yield sodium chloride crystals that constitute common salt. Sea water is not just a solution of sodium chloride and water, but rather is a mixture of virtually every element on Earth. Anything that can be washed downstream eventually finds its way to the seas, and is incorporated into the solution of the oceans [9].

The relatively high abundance of 'essential' elements in humans, bacteria and sea water in Fig. 2 suggests that terrestrial life does not have elemental preferences and is based on the most abundant elements in the environment. Additionally we observe that the composition of life is not exactly the same as its environment. Some elements like carbon, nitrogen and phosphorus are more abundant in life than in sea water while other elements like chlorine and sodium are more abundant in seawater than in life. An increased abundance of an element in life, relative to its abundance in sea water (for example carbon and phosphorus in Fig. 2), is a measure of life's concentration of the element from the environment. Elements like tungsten or uranium, which are not regarded as 'essential' to life appear to have similar abundances in life and in sea water and appear along the diagonal line in Fig. 2. It is tempting to suggest that life concentrates 'essential' elements from its environment while 'non-essential' elements occur in equilibrium abundances in life and its environment. However, a number of elements not 
regarded as 'essential;' like aluminium, germanium and cerium, are also in high abundance in life relative to sea water.

We also observe that elements recognised as 'essential' are not necessarily concentrated in life like sodium and magnesium which are more abundant in sea water. Some elements may not be 'essential' for an organism but are concentrated in the organism as a consequence of sequestration of another element. For example, $\mathrm{Pb}^{2+}$ ions have similar binding preferences to $\mathrm{Ca}^{2+}$ and $\mathrm{Zn}^{2+}$ ions in the metal centres of proteins as they all have similar effective ionic charge to radius ratios. It is difficult for metabolic pathways to distinguish between ions of these elements and the toxic effects of lead poisoning are attributed to displacement of $\mathrm{Ca}^{2+}$ and $\mathrm{Zn}^{2+}$ ions by $\mathrm{Pb}^{2+}$ ions in metalloenzymes [10,11]. In humans, lead is found incorporated into the mineral matrix of calcium rich bones.

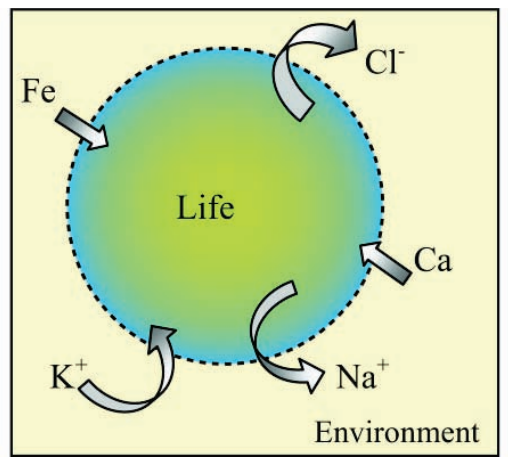

Fig. 3: Life pulls in some elements like iron and calcium from the environment while it pushes out elements like sodium and chlorine.

These observations lend support to the idea that life is a system which manipulates elemental abundances away from their equilibrium abundances in its environment, to perform its metabolic activities, ultimately to harness energy. Imagine that life begins as a bag containing the same elements in the same proportion as in the external environment. Then life somehow learns to pull in some elements from the outside world and concentrates them inside the bag and push out other elements as illustrated in Fig. 3. We can now define life as a chemical system away from equilibrium with its environment. The disequilibrium established by the unequal abundance of an element in life, relative to its environment is used by life to perform its metabolic activities.

We are not suggesting that organisms are bags of independent elements. Organisms are chemical entities and are produced, maintained, and propagated by chemical reactions, in the form of highly complex coupled networks, which are the product of evolution over the past approximately $3.5-4$ billion years.

We plan to perform elemental analyses of various species using the analytical techniques that offer much higher precision than ever before and hope to remedy the lack of quality data for other species (not just humans and bacteria). Widespread studies looking at all forms of life on Earth would be required to work towards our ultimate goal of understanding the origins and evolution of life. However, given that our approach is at an early stage, we must have a modest realisable target that will eventually help us assemble the 'big picture'. This will be achieved by focusing on the root of the phylogenetic tree commonly described as the Tree of Life illustrated in Fig. 4. We plan to study the elemental composition of the archean and bacterial species that have been the dominant life form for most of life's existence on the Earth ${ }^{8}$ and are more closely related to the Last Universal Common Ancestor (LUCA) than eucaryotes. Many of the extant archean and bacterial species were present on the early Earth and are found today in extremely acidic or hot environments.

The chemical composition of an organism may be a highly conserved feature of terrestrial life allowing us to relate deeply branched extant species by their elemental composition and in turn infer the composition of the Last Universal Common Ancestor and its environment. Such knowledge will further our understanding of the possible sites for the origin and early evolution of life on Earth.

Using branch lengths as a proxy for time, analyses of the elemental composition of species with various branch lengths across the phylogenetic tree will be used to determine if there is any identifiable trend of life using a larger suite of elements as a function of time. This approach also lends itself to identifying evolutionary steps linked to changes in the bioavailability of elements. For example, oxygenation of the atmosphere approximately 2.34 billion years ago caused substantial decrease in

\footnotetext{
${ }^{8}$ Although controversial isotopic evidence by [13] suggests the presence of autotrophic organisms 3.7 billion years ago, bacteria have existed for at least 3.4 billion years, as indicated by stromatolite fossils [14] and microbial life was the only form of life until 2 billion years ago. Animals appeared 600 million years ago and the Homo genus that includes modern humans has existed for 2.5 million years.
} 


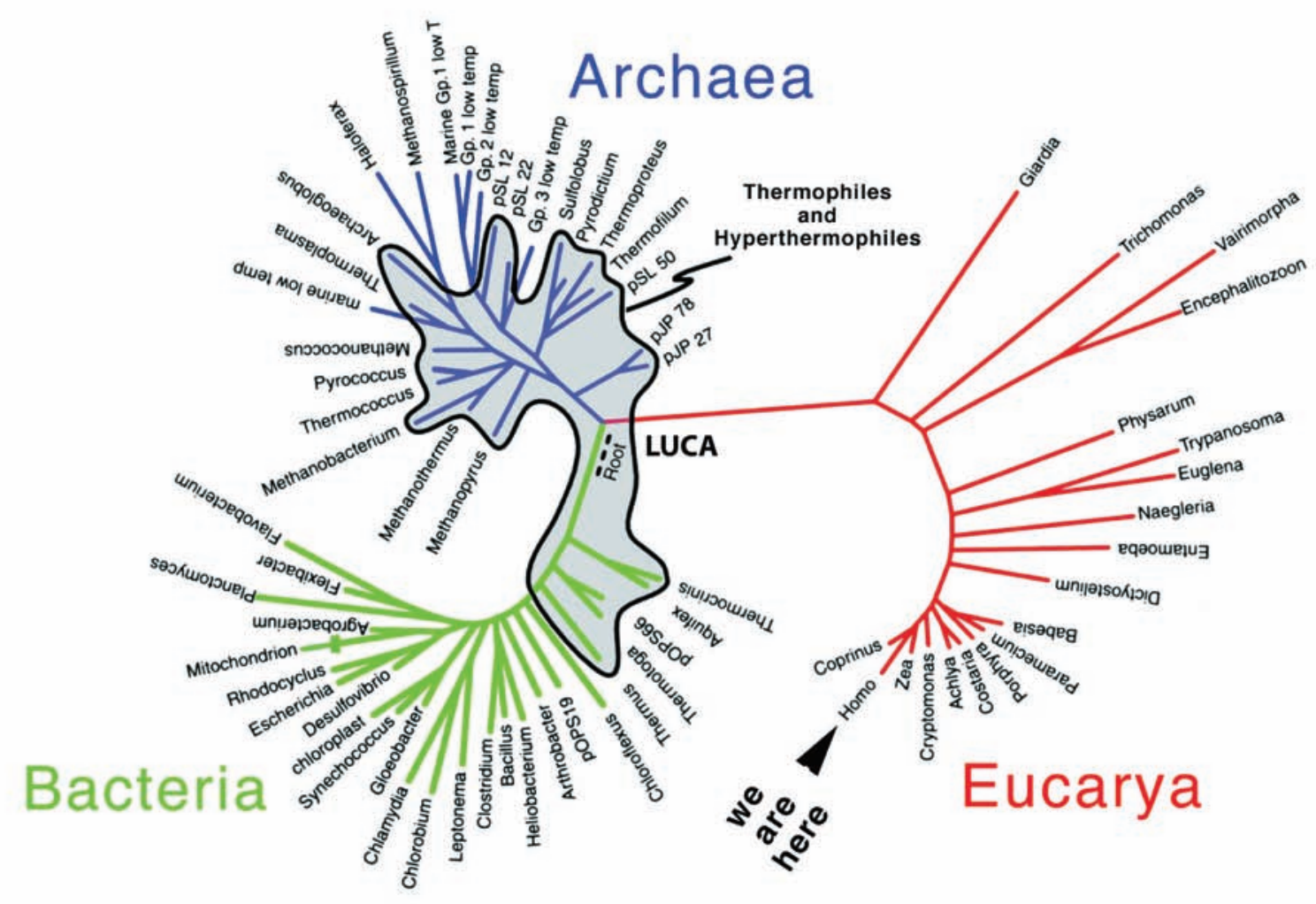

Fig. 4: The Phylogenetic Tree of Life based on comparisons of 16s rRNA sequence of extant organisms [12].

the availability of the biologically active form of iron in the oceans (from soluble $\mathrm{Fe}^{2+}$ to $\mathrm{Fe}^{3+}$ which forms insoluble precipitates of $\left.\mathrm{Fe}(\mathrm{OH})_{3}\right)$. The oxygenation also oxidised another biologically active metal, copper from its $\mathrm{Cu}^{+}$to $\mathrm{Cu}^{2+}$ state [15]. [16] have suggested that this change in the environment may have triggered the development of new metabolic pathways which harnessed the electrochemical potential of the higher oxidation state of copper often in roles previously performed by iron.

Advancements in molecular biology, bioinformatics and elemental analysis over the last decade means that we now have the tools to perform our investigations successfully. [1] observed the similarities in the elements that make up life and its environment and reasoned that "... man was created from the dust of the Earth." 150 years on, we are examining both the similarities and the differences in the elements that make up life and its environment and our interpretations will allow us to better understand the origins and the evolution of life. Answering questions like 'What is life made of?' will help us answer one of man's most fundamental questions: 'What is life?'

\section{ACKNOWLEDGMENTS}

This article is a shortened version intended for a university-wide audience, of a more detailed conference proceedings article [17].

\section{REFERENCES}

[1] R. De Courcey, Man displayed - in four parts, Hamilton, Ontario, 1857

[2] R. J. P. Williams and J. J. R. Fraústo da Silva, Fitness of the Cosmos for Life: Biochemistry and Fine-Tuning, chapter Evolution revisited by inorganic chemists, pp. 456-489, Cambridge University Press, Cambridge, 2007
[3] W. S. Snyder, M. J. Cook, I. H. Tipton,

E. S. Nasset, L. R. Karhausen and G. P. Howells, Reference Man: Anatomical, Physiological and Metabolic Characteristics, Vol. 23 of Report of Task Group on Reference Man - International Commission on Radiological Protection, Pergamon Press Ltd., New York, $1^{\text {st }}$ edition, 1975 
[4] J. R. Porter, Bacterial Chemistry and Physiology, John Wiley and Sons, London, 1946

[5] R. E. Davies and R. H. Koch, Philosophical Transactions: Biological Sciences, 334(1271):391-403, 1991

[6] H. J. M. Bowen, Environmental Chemistry of the Elements, Academic Press Inc., London, 1979

[7] J. P. Riley and G. Skirrow, Chemical Oceanography, Academic Press Inc., London, 1989

[8] B. L. Oser, Hawk's Physiological Chemistry, McGraw-Hill Book Company, Sydney, $14^{\text {th }}$ edition, 1965

[9] M. E. Q. Pilson, An Introduction to the Chemistry of the Sea, Prentice-Hall Inc., New Jersey, 1998

[10] E. Nieboer, G. G. Fletcher and Y. Thomassen, Journal of Environmental Monitoring, 1:1-14, 1999

[11] H. A. Godwin, Current Opinion in Chemical Biology, 5(2):223-227, 2001
[12] Online. Accessed 12 December 2008, $<$ http://www.astro.washington.edu/endsofworld/>

[13] M. T. Rosing, Science, 283(5402):674-676

[14] A. C. Allwood, M. R. Walter, B. S. Kamber, C. P. Marshall and I. W. Burch, Nature, 441:714718, 2006

[15] H. J. Schellnhuber, E. Szathmary and T. M. Lenton, Nature, 431(7011):913, 2004

[16] R. J. P. Williams and J. J. R. Fraústo da Silva, Journal of Theoretical Biology, 220(3):323-343, 2003

[17] A. Chopra and C. H. Lineweaver, 2009 in Australian Space Science Conference Series: $8^{\text {th }}$ Conference Proceedings, NSSA Full Referee, Proceedings DVD, $1^{\text {st }}$ ed, National Space Society of Australia Ltd, edt. W. Short \& I. Cairns, Conference held in Canberra, Australia $29^{\text {th }}$ September $-1^{\text {st }}$ October, 2008, ISBN 13:978-09775740-2-5 